\title{
Ernährung \\ Nahrungscholesterin - künftig nicht mehr der Schurke in der Ernährung?
}

Nach fünf Jahrzehnten der Verteufelung kommt es wohl in den USA zu einer neuen Bewertung des über die Nahrung aufgenommenen Cholesterins, schreibt Chris Weller auf Medical Daily. Im Jahr 1961 veröffentlichte die American Heart Association (AHA) eine Studie, die das Verhältnis (nicht nur) der Amerikaner zu Cholesterin über Jahrzehnte beeinflussen sollte. Im Überschuss aufgenommenes Cholesterin, so lernten es die diätbewussten Bürger, könne zur Koronarerkrankung und Herzattacken führen, kurz: Cholesterin war der Feind. Der Verzehr von Nahrungsmitteln wie Butter, Käse und Eiern litt massiv unter diesem Angriff. Daten des US-Agrarministeriums belegen, dass seit Veröffentlichung dieser Studie z. B. der Eierverzehr jährlich gefallen ist. Aßen die Menschen 1945 im Durchschnitt noch 421,4 Eier, so waren es 2010 weniger als 250. Seither gab es keine Evidenz, die stark genug war, die Empfehlungen infrage zu stellen. Jetzt aber veröffentlicht die staatliche Kommission für Ernährungsrichtlinien neue Empfehlungen, die nahelegen, dass die bisherigen Leitlinien nicht nur fehlgeleitet, sondern sogar schlichtweg falsch waren.
Der Kardiologe Steve Nissen erklärt dazu, dass „wir über die Jahre und Jahrzehnte hinweg falsche Diätrichtlinien hatten“. Schließlich haben wir gelernt, dass Cholesterin tatsächlich für viele Körperprozesse essenziell ist, Zellmembranen stabilisiert und bei der Synthese der Gallensäure und der Verarbeitung von Vitamin D unterstützt. In den vergangenen Jahren wurden die Menschen davor gewarnt, täglich mehr als 300 mg Cholesterin aufzunehmen - grob geschätzt ist diese Menge in einem Ei enthalten. Die neuen Leitlinien besagen jetzt, dass nur Menschen mit Krankheiten wie Diabetes oder einem genetisch hohen Cholesterinspiegel (familiäre Hypercholesterinämie) sich darüber Sorgen machen müssten, dass das über die Nahrung aufgenommene Cholesterin ihren Cholesterinspiegel insgesamt negativ beeinflussen könnte. Die neuen Leitlinien sollen jetzt auch geänderte Empfehlungen zur Aufnahme von Salz, Zucker, rotem Fleisch, gesättigten Fettsäuren und Omega-3-Fettsäure enthalten. „Nahezu zu jedem einzelnen Nährstoff liegen mittlerweile peer-reviewte Studien mit nahezu jedem denkbaren Ergebnis vor“, erklärt dazu Prof. John P.A. Ioannidis von der Stanford Uni-

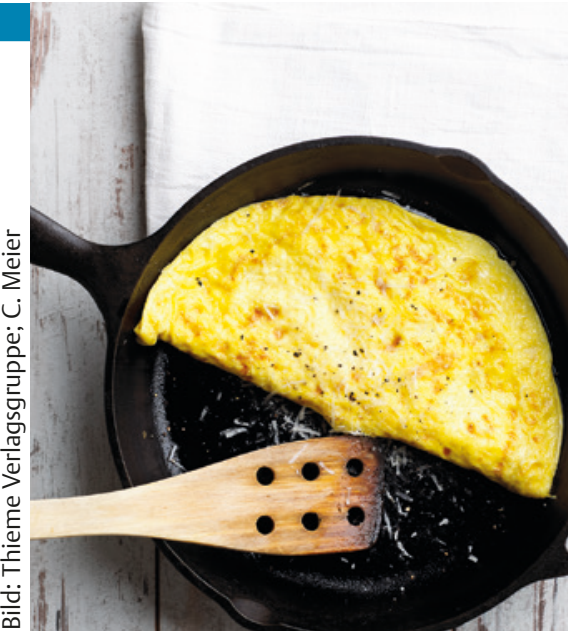

versity. Und er erklärt: „Bei dieser Epidemie der Studien und Daten - auf welche will man sich denn verlassen und welche Ergebnisse sind korrekt?"

Steve Nissen, der immer gerne auch kontrovers diskutiert, erklärt, dass die neuen Leitlinien endlich vielleicht beim Kampf gegen die Adipositas-Epidemie helfen könnten, denn die Hinwendung zu Zucker und Kohlenhydraten und die darauf folgende Zunahme der Diabeteserkrankungen resultiere aus der Furcht der Amerikaner vor dem Fett. In Wahrheit, so Nissen, könne es sein, dass die Lösung gefährlicher gewesen sei als es das Problem jemals war. Günther Buck, Weilheim

Quelle

Medical Daily, 11. Februar 2015

\section{Bessere Ergebnisse bei insulinpflichtigen Patienten durch eine Pumpe?}

Während der Nutzen einer Insulinpumpe bei Patienten mit Typ-1-Diabetes recht klar ist, wird ihr Einsatz bei insulinpflichtigen Typ-2-Patienten häufig noch diskutiert. Man schätzt, dass weltweit rund 20 Millionen Patienten mit Typ-2-Diabetes Insulin benötigen. Viele dieser Patienten, die Rede ist von rd. 57\%, erreichen aber auch mit mehrfachen täglichen Insulininjektionen keine gute glykämische Kontrolle. Diese Patienten sind gefährdet und schwer zu führen, häufig leiden sie auch unter Komplikationen. Eine pumpengesteuerte Insulinabgabe kann für diese Gruppe von hohem Nutzen sein.

Die OpT2mise-Studie, in die 331 Patienten im Alter zwischen 30 und 75 Jahren aufgenommen wurden (168 erhielten eine Pumpe, 163 multiple tägliche Insulininjektionen), untersuchte den Nutzen einer pumpengesteuerten Insulinverabreichung [1]. Der Ausgangswert des $\mathrm{HbA}_{1 \mathrm{c}}$ lag in beiden Gruppen bei $9 \%$. Bei den Patienten mit einer Pumpe (MiniMed Insulin-Pumpe) erniedrigte sich der $\mathrm{HbA}_{1 \mathrm{c}}$-Spiegel um 1,1 vs. $0,4 \%$ bei den Patienten mit multiplen täglichen Insulininjektionen $(\mathrm{p}<0,001)$. Bei den Pumpenpatienten traten keine schwerwiegenden hypoglykämischen Episoden oder Ketoazidosen auf und ihre tägliche Insulindosis war um 20,4\% niedriger $(p<0,001)$. Die Gewichtsveränderungen in den beiden Gruppen unterschieden sich jedoch nicht. Der Anteil der Patienten mit einem $\mathrm{HbA}_{1 \mathrm{c}}<8 \%$ war in der
Pumpengruppe doppelt so hoch wie in der Gruppe mit multiplen täglichen Insulininjektionen (55 vs. 28\%). Im Rahmen des EASD 2014 wurden drei weitere Poster zur Studie veröffentlicht, die weiter eine hohe Zufriedenheit und bessere Einstellung der Patienten mit der Pumpentherapie zeigen, und zwar unabhängig vom Alter, Diabetesdauer, kognitivem Zustand der Patienten und der Compliance bei der Blutzuckermessung.

Günther Buck, Weilheim

Quelle

1 Reznik $Y$ et al. Insulin pump treatment compared with multiple daily injections for treatment of type 2 diabetes (OpT2mise): a randomised open-label controlled trial. The Lancet 2014; 384 (9950): 1265-1272 


\section{Typ-2-Patienten mit nur leicht erhöhtem Blutdruck profitieren weniger}

Unbestritten reagieren Patienten mit erhöhtem Blutdruck und Typ-2-Diabetes durch die Absenkung des erhöhten Druckes mit einem geringeren Risiko für die Entwicklung kardiovaskulärer Probleme, stellt eine gerade veröffentlichte Metaanalyse fest [1].

Diese Metaanalyse umfasste 40 Einzelstudien im Zeitraum zwischen 1966 und 2014 mit insgesamt mehr als 100000 Patienten mit erhöhtem Blutdruck und Typ-2-Diabetes, die mit Diuretika, ACE-Hemmern und weiteren den Blutdruck senkenden Medikamenten behandelt wurden. Als „normal“ wurde ein Druck von 120/80 mmHg eingestuft, bis $140 / 90 \mathrm{mmHg}$ galt er als „prähypertensiv“, darüber lag ein Hochdruck vor.

Für jede Absenkung des systolischen Drucks um $10 \mathrm{mmHG}$ sank das Sterberisiko in der Metaanalyse um etwa $10 \%$, auch wurde das Risiko für ein akutes kardiovaskuläres Ereignis, für Schlaganfall und die Entwicklung einer diabetischen Retinopathie gesenkt: Relatives Risiko (RR), 0,87; 95\% CI, 0,78-0,96); absolute Risikoreduktion (ARR) als Ereignisse pro 1000 Patientenjahre $(3,16$; $95 \%$ CI, 0,90$5,22)$, kardiovaskuläre Ereignisse (RR, 0,89 [95\% CI, 0,83-0,95]; ARR, 3,90 [95\% CI, 1,57-6,06]), KHK (RR, 0,88 [95\% CI,
0,80-0,98]; ARR, 1,81 [95\% CI, 0,353,11]), Schlaganfall (RR, 0,73 [95\% CI, 0,64-0,83]; ARR, 4,06 [95\% CI, 2,535,40]), Albuminurie (RR, 0,83 [95\% CI, 0,79-0,87]; ARR, 9,33 [95\% CI, 7,1311,37]), und Retinopathie (RR, 0,87 [95\% CI, 0,76-0,99]; ARR, 2,23 [95\% CI, 0,15$4,04])$. Zwar profitierten insgesamt alle Patienten, bemerkenswert war aber, dass sich das Risiko am stärksten bei den Patienten verringerte, deren systolischer Ausgangsblutdruck über $140 \mathrm{mmHg}$ lag, so die Autoren der Studie. Zieht man die Nebenwirkungen der für die Senkung des Blutdrucks eingesetzten Präparate in Betracht - so können beispielsweise Diuretika den Blutzucker bei Diabetikern sogar erhöhen - sollte bei Patienten mit Werten unter 140/90 mmHg deren Nutzen und potenzieller Schaden gegeneinander abgewogen werden.

Zwar wurden auf der Grundlage einer USamerikanischen Studie die Zielwerte für diabetischen Patienten weniger strikt gefasst, teils wurde ein abwartendes Verhalten bis zu einem Wert von 140/90 mmHg empfohlen - dies sei aber ein wenig zu lasch, erklärte Dr. Bryan Williams vom University College in London in einem begleitenden Editorial. Er vertritt die Auffassung, dass es bei einer Empfehlung auf

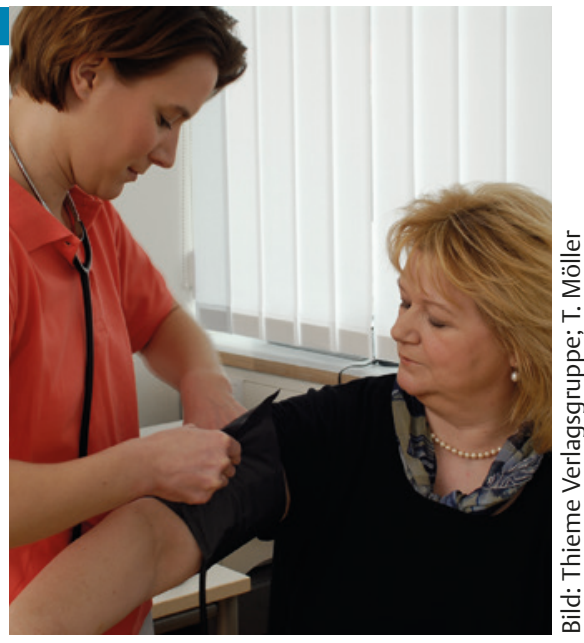

Absenkung des Blutdrucks in einen Bereich unter 140/80 $\mathrm{mmHg}$ bleiben sollte, besser noch wäre ein Zielwert von unter $130 / 80 \mathrm{mmHg}$, wenn der Patient dies toleriert. Es gebe keine magische Schwelle, über der der Blutdruck plötzlich schädlich wird oder unter der er vorteilhaft ist, meint der Experte. Die Grenzwerte seien schlicht und einfach dazu eingeführt worden, die Durchführung von Studien zu erleichten und Richtlinien zu erstellen, aber in Wirklichkeit sei das Risiko fließend, eine definitive Schwelle dafür existiere nicht.

Günther Buck, Weilheim

Quelle

1 Emdins $C$ et al. Blood Pressure Lowering in Type 2 Diabetes. A Systematic Review and Metaanalysis. JAMA 2015; 313 (6): 603-615 doi:10.1001/jama.2014.18574

\section{Studien}

\section{Ob Erfolg oder Fehlschlag - Studien sollen publiziert werden}

Immer wieder werden Teilergebnisse von Studien bekannt gegeben, ohne dass man jemals die den Ergebnissen zugrunde liegenden Ergebnisse nachlesen kann. Bestenfalls steht dann als Quelle der Zusatz „data on file“. Die staatlichen Behörden in den USA wollen dies ändern: Studien über Medikamente und medizinische Geräte sollen publiziert werden, unabhängig davon, ob sie erfolgreich oder ein Fehlschlag waren. Und dies soll dann auch für Tausende von alten Studien gelten. Der vom Department of Health and Human Services und dem National Institutes of Health (NIH) ausgearbeitete Vorschlag würde die Hersteller noch nicht zugelassener Medikamente und Geräte zwingen, Zusam- menfassungen der Studienergebnisse spätestens nach einem Jahr auf der Regierungsseite ClinicalTrials.gov zu publizieren. Bisher gilt dies nur für zugelassene Produkte und das sind nach dem NIH nur rund 15000 von 178000 Studien. Phase-1- und Machbarkeits-Studien sollen von dieser Regelung ausgenommen bleiben, und die Regelung hätte auch keine Auswirkungen auf das Design der Studien, die für die Zulassung durch die FDA erforderlich sind. Sie würde aber den öffentlichen Pool an klinischen Ergebnissen erheblich erweitern und helfen, doppelte Studien zu vermeiden und unsichere und nicht wirksame Produkte auszusortieren.
In der Zwischenzeit haben einige der großen Arzneimittelhersteller bereits nachgegeben und stellen in unterschiedlichem Ausmaß Daten auf ClinicalStudyDataRequest.com zur Verfügung. Einige, wie beispielsweise Sanofi und Bayer, stellen nur Ergebnisse neu zugelassener Produkte zur Verfügung, während andere wie GlaxoSmithKlinie und Johnson \& Johnson zugestimmt haben, im Interesse der globalen Forschung große anonymisierte Datenmengen zur Verfügung zu stellen.

Günther Buck, Weilheim

\section{Quelle}

Fierce Biotech (Biotech Industries Daily Monitor), 14. November 2015 